\title{
Catalytic Light-Driven Generation of Hydrogen from Water by Iron Dithiolene Complexes
}

\author{
Hongjin Lv, T. Purnima A. Ruberu, Valerie E. Fleischauer, William W. Brennessel, Michael L. Neidig, Richard \\ Eisenberg* \\ Department of Chemistry, University of Rochester, Rochester, New York 14627, USA
}

Corresponding author e-mail: eisenberg@chem.rochester.edu

\section{Table of contents}

Ligand exchange to make water-soluble CdSe QDs

Quantification of capping ligands on the surface of CdSe QDs

Fig. S1.ORTEP diagrams of 1

Table S1. Crystallographic data for $\mathbf{1}$

Fig. S2.ORTEP diagrams of 2

Table S2. Crystallographic data for 2

Fig. S3.ORTEP diagrams of $\mathbf{3}$

Table S3. Crystallographic data for $\mathbf{3}$

Fig. S4.ORTEP diagrams of $\mathbf{4}$

Table S4. Crystallographic data for 4

Table S5. Redox potentials for Fe-complexes 1-4

Fig. S5. Cyclic voltammograms of $0.5 \mathrm{mM}$ Fe-dithiolene complexes with TFA

Fig. S6.UV-vis spectra of CdSe NCs with different capping agents

Fig. S7. UV-vis spectral change of complexes 1-4 solution on addition of DHLA

Fig. S8. UV-vis spectral change of complexes 2 solution with varying DHLA

Chart S1. Control experiments of hydrogen evolution

Fig. S9. Hydrogen production by varying [catalyst 2] concentration

Fig. S10. Hydrogen production by varying [CdSe-S3] concentration

Fig. S11. Hydrogen production by varying [AA] concentration

Fig. S12. Effect of addition of DHLA to the systems containing CdSe-S3/Fe( $\left(\mathrm{NO}_{3}\right)_{3} / \mathrm{AA}$

Fig. S13. Effect of addition of DHLA to the systems containing CdSe-S3/1-4/ AA

Fig. S14. Comparison of activity between $\mathrm{Fe}\left(\mathrm{NO}_{3}\right)_{3}$ and $\mathbf{3}$ in the presence of DHLA using $\mathrm{CdSe}-\mathrm{S} 3 \mathrm{NCs}$ photosensitizers

Table S6. TONs per Fe atom for $\mathrm{H}_{2}$ production using complex 2 or $\mathrm{Fe}\left(\mathrm{NO}_{3}\right)_{3}$ with varying DHLA

Fig. S15. UV-vis spectra of CdSe NCs before and after photolysis

Fig. S16. Hydrogen production using isolated CdSe-DHLA photosensitizer

Fig. S17. Hydrogen production using isolated CdSe-S3 photosensitizer

Table S7. Hydrogen production data using different Fe-catalysts and CdSe-S3 NCs 
Fig. S18. Hydrogen production using CdSe-DHLA with varying amount of DHLA capping units

Table S8. Hydrogen production data using different Fe-catalysts and CdSe-DHLA NCs

Fig. S19. Hydrogen production using CdSe-MUA NCs/2/AA with or without DHLA

\section{Ligand exchange to make water-soluble CdSe QDs.}

Synthesis of water-soluble CdSe-DHLA and CdSe-S3 QDs.The water-soluble CdSe QDs were prepared following a modified literature method. ${ }^{1,2}$ In a typical experiment, a total of $600 \mu \mathrm{L}$ of $\sim 25 \%$ tetraethylammonium hydroxide solution in methanol was added to $15 \mathrm{~mL}$ of methanol containing $200 \mathrm{nmol}$ of CdSe-TOPO under a stream of $\mathrm{N}_{2}$. To this mixture, $100 \mu \mathrm{L}$ of DHLA (for the synthesis of CdSe-DHLA QDs) or $80 \mathrm{mg}$ 3-mercapto-2,2-bis(mercaptomethyl)propanoic acid (for the synthesis of CdSe-S3 QDs) dissolved in $5 \mathrm{~mL}$ dry methanol was added. Then, the mixture was refluxed for $6 \mathrm{hrs}$ under $\mathrm{N}_{2}$ atmosphere. The resulting CdSe-DHLA or CdSe-S3 QDs were precipitated from the methanolic solution by the addition of diethyl ether and collected by centrifugation $(10,000 \mathrm{RPM})$. This procedure was repeated one more time to remove all unreacted ligand residues. The washed CdSe-DHLA or CdSe-S3 QDs were suspended in deionized water and stored under ambient conditions in dark.

Synthesis of water-soluble CdSe-MUA QDs. The CdSe-MUA QDs were prepared in a procedure analogous to that described CdSe-DHLA or CdSe-S3 QDs except that reactant quantities were changed. In this preparation, $50 \mathrm{nmol}$ of $\mathrm{CdSe}-\mathrm{TOPO}, 400 \mu \mathrm{L}$ of $25 \%$ tetraethylammonium hydroxide solution in methanol, and $33.5 \mathrm{mg}$ MUA ligands were used.

Synthesis of water-soluble CdSe-MPA QDs. TheCdSe-MPA QDs were prepared in a procedure analogous to that described CdSe-DHLA or CdSe-S3 QDs but $50 \mathrm{nmol}$ of CdSeTOPO, $1000 \mu \mathrm{L}$ of $\sim 25 \%$ tetraethylammonium hydroxide solution in methanol, and $30 \mu \mathrm{L}$ MPA ligands were used. Also, the resulting mixture was refluxed for $14 \mathrm{hrs}$ under $\mathrm{N}_{2}$ to ensure complete ligand exchange.

Quantification of capping ligands on the surface of CdSe QDs. The extents of capping ligands on the surface of CdSe QDs were quantified by ${ }^{1} \mathrm{H}$ NMR spectroscopy. The detailed experimental procedure follows modified literature method. ${ }^{3}$ Generally, the $\mathrm{pH}$ of CdSe-DHLA or $\mathrm{CdSe}-\mathrm{S} 3$ solution was adjusted to 0 by addition of $\mathrm{HCl}$ solution $(6 \mathrm{M})$, the mixture was degassed well and kept in dark for 15 hours. During this period, the CdSe QDs were precipitated and removed by centrifugation. The resulting supernatant was then dried at $\sim 45{ }^{\circ} \mathrm{C}$ heating and the obtained solid was re-dissolved in $0.8 \mathrm{~mL}$ DMSO- $d_{6}$ containing $750 \mu \mathrm{M}$ benzene as an internal standard. The ${ }^{1} \mathrm{H}$ NMR spectrum was recorded on a Bruker Avance $400 \mathrm{MHz}$ spectrometer. The concentration of the DHLA or S3 capping agents was calculated based on peak integrations relative to benzene; and the molar ratio of DHLA or S3 to CdSe QDs was used to denote different CdSe QDs light-absorbers (e.g. 100 S3 units per CdSe dot indicates the ratio of $\mathrm{S} 3$ capping agents per CdSe dot is 100). 

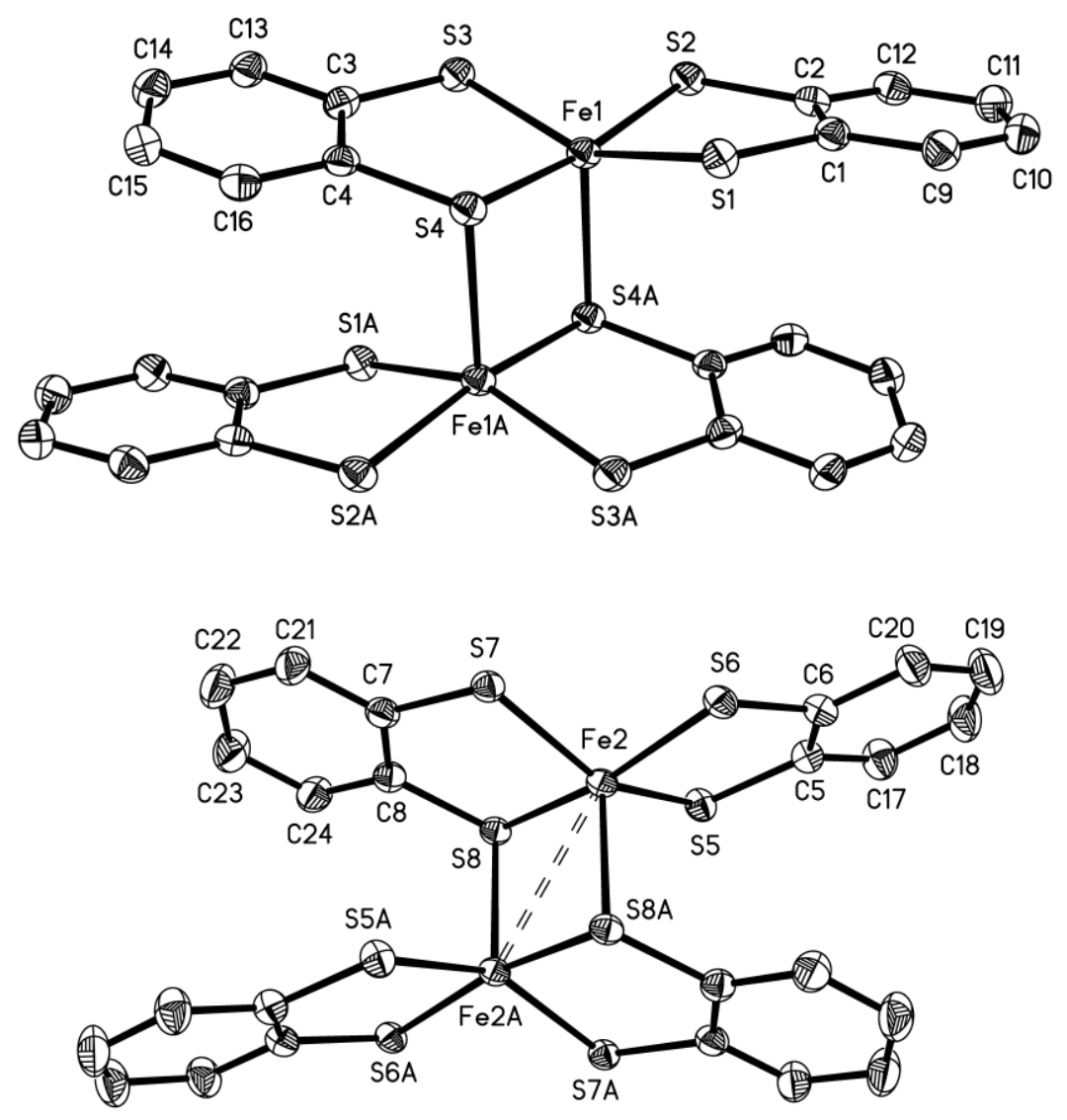

Fig. S1. ORTEP diagram of 1 (up: 1a; down: 1b).

Table S1. Crystallographic data and structure refinement for $\mathbf{1 .}$

\begin{tabular}{lll}
\hline Empirical formula & $\mathrm{C}_{56} \mathrm{H}_{88} \mathrm{Fe}_{2} \mathrm{~N}_{2} \mathrm{~S}_{8}$ & \\
Formula weight & 1157.46 \\
Temperature & $100.0(5) \mathrm{K}$ \\
Wavelength & $0.71073 \AA$ \\
Crystal system & triclinic & \\
Space group & $P 1$ & \\
Unit cell dimensions & $a=12.6555(7) \AA$ & $\alpha=85.1546(12)^{\circ}$ \\
& $b=15.1068(9) \AA$ & $\beta=88.2286(13)^{\circ}$ \\
& $c=16.1136(9) \AA$ & $\gamma=75.2419(12)^{\circ}$ \\
Volume & $2968.2(3) \AA 3$ & \\
$Z$ & 2 & \\
Density (calculated) & $1.295 \mathrm{Mg} / \mathrm{m}^{3}$ & \\
Absorption coefficient & $0.807 \mathrm{~mm}^{-1}$ & \\
$F(000)$ & 1236 & \\
Crystal color, morphology & black, plate & \\
\hline
\end{tabular}


Crystal size

Theta range for data collection

Index ranges

Reflections collected

Independent reflections

Observed reflections

Completeness to theta $=33.729^{\circ}$

Absorption correction

Max. and min. transmission

Refinement method

Data / restraints / parameters

Goodness-of-fit on $F^{2}$

Final $R$ indices $[I>2 \operatorname{sigma}(I)]$

$R$ indices (all data)

Largest diff. peak and hole
$0.25 \times 0.20 \times 0.12 \mathrm{~mm}^{3}$

1.883 to $33.768^{\circ}$

$-19 \leq h \leq 19,-23 \leq k \leq 23,-25$

$\leq l \leq 25$

60914

$23557[R($ int $)=0.0618]$

14403

$99.2 \%$

Multi-scan

0.7467 and 0.6741

Full-matrix least-squares

on $F^{2}$

23557 / 0 / 621

0.932

$R 1=0.0551, w R 2=0.1174$

$R 1=0.1000, w R 2=0.1373$

1.373 and -0.441 e. $\AA^{-3}$

Some equations of interest:

$$
\begin{aligned}
& R_{\text {int }}=\Sigma\left|F_{\mathrm{O}}^{2}-\left\langle F_{\mathrm{O}}^{2}\right\rangle\right| / \Sigma\left|F_{\mathrm{O}}{ }^{2}\right| \\
& R 1=\Sigma\left\|F_{\mathrm{O}}|-| F_{\mathrm{c}}\right\| / \Sigma\left|F_{\mathrm{O}}\right| \\
& w R 2=\left[\Sigma\left[w\left(F_{\mathrm{O}}^{2}-F_{\mathrm{c}}{ }^{2}\right)^{2}\right] / \Sigma\left[w\left(F_{\mathrm{O}}{ }^{2}\right)^{2}\right]\right]^{1 / 2} \\
& \text { where } w=1 /\left[\sigma^{2}\left(F_{\mathrm{O}}^{2}\right)+(a P)^{2}+b P\right] \text { and } \\
& P=1 / 3 \max \left(0, F_{\mathrm{O}}^{2}\right)+2 / 3 F_{\mathrm{c}}^{2} \\
& \mathrm{GOF}=S=\left[\Sigma\left[w\left(F_{\mathrm{O}}^{2}-F_{\mathrm{c}}{ }^{2}\right)^{2}\right] /(m-n)\right]^{1 / 2}
\end{aligned}
$$

where $m=$ number of reflections and $n=$ number of parameters

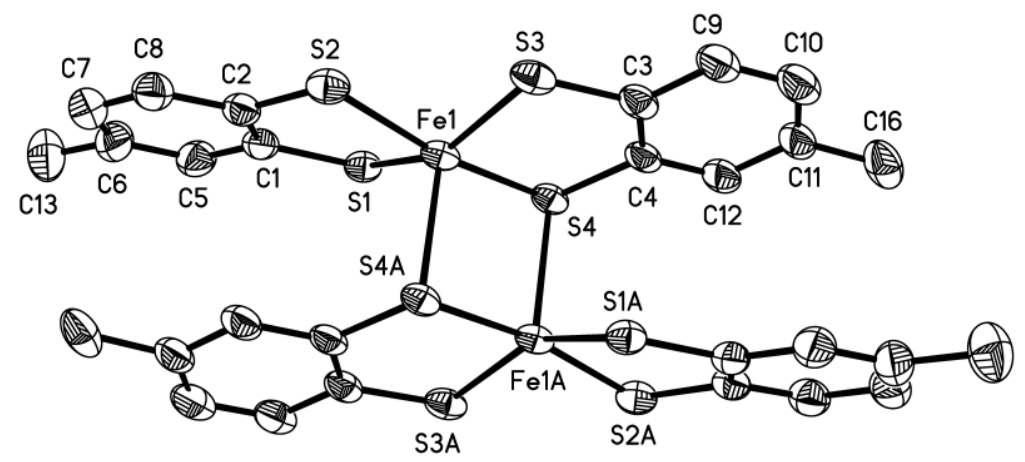

Fig. S2. ORTEP diagram of 2.

Table S2. Crystallographic data and structure refinement for 2. 
Empirical formula

Formula weight

Temperature

Wavelength

Crystal system

Space group

Unit cell dimensions

Volume

Z

Density (calculated)

Absorption coefficient

$F(000)$

Crystal color, morphology

Crystal size

Theta range for data collection

Index ranges

Reflections collected

Independent reflections

Observed reflections

Completeness to theta $=25.028^{\circ}$

Absorption correction

Max. and min. transmission

Refinement method

Data / restraints / parameters

Goodness-of-fit on $F^{2}$

Final $R$ indices $[I>2 \operatorname{sigma}(I)]$

$R$ indices (all data)

Largest diff. peak and hole
$\mathrm{C}_{60} \mathrm{H}_{96} \mathrm{Fe}_{2} \mathrm{~N}_{2} \mathrm{~S}_{8}$

1213.56

$100.0(5) \mathrm{K}$

$0.71073 \AA$

trigonal

$R-3$

$a=34.115(2) \AA$

$\alpha=90^{\circ}$

$b=34.115(2) \AA$

$\beta=90^{\circ}$

$c=15.1763(9) \AA$

15296(2) $\AA^{3}$

9

$1.186 \mathrm{Mg} / \mathrm{m}^{3}$

$0.708 \mathrm{~mm}^{-1}$

5850

black, plate

$0.30 \times 0.12 \times 0.08 \mathrm{~mm}^{3}$

1.924 to $25.269^{\circ}$

$-40 \leq h \leq 40,-40 \leq k \leq$

$40,-17 \leq l \leq 18$

42729

$6175[R($ int $)=0.0902]$

3444

$100.0 \%$

Multi-scan

0.7452 and 0.6842

Full-matrixleast-squares

on $F^{2}$

6175 / 66 / 382

1.010

$R 1=0.0588, w R 2=0.1460$

$R 1=0.1164, w R 2=0.1829$

0.559 and -0.293 e. $\AA^{-3}$ 


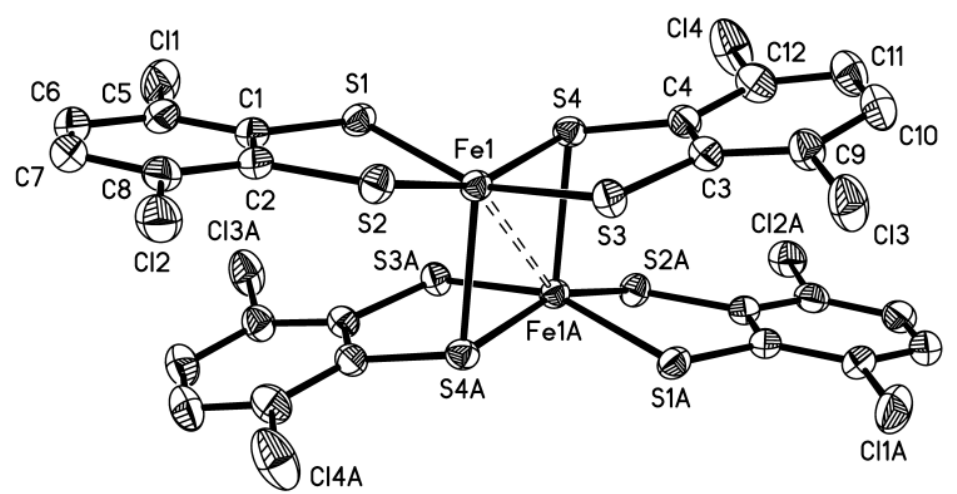

Fig. S3. ORTEP diagram of 3.

Table S3. Crystallographic data and structure refinement for 3.

\begin{tabular}{|c|c|c|}
\hline Empirical formula & $\mathrm{C}_{56} \mathrm{H}_{80} \mathrm{Cl}_{8} \mathrm{Fe}_{2} \mathrm{~N}_{2} \mathrm{~S}_{8}$ & \\
\hline Formula weight & 1433.00 & \\
\hline Temperature & $100.0(5) \mathrm{K}$ & \\
\hline Wavelength & $0.71073 \AA$ & \\
\hline Crystal system & monoclinic & \\
\hline Space group & $P 2_{1} / n$ & \\
\hline \multirow[t]{3}{*}{ Unit cell dimensions } & $a=14.0308(12) \AA$ & $\alpha=90^{\circ}$ \\
\hline & $b=16.9507(14) \AA$ & $\beta=103.7780(18)^{\circ}$ \\
\hline & $c=14.2666(12) \AA$ & $\gamma=90^{\circ}$ \\
\hline Volume & $3295.4(5) \AA^{3}$ & \\
\hline$Z$ & 2 & \\
\hline Density (calculated) & $1.444 \mathrm{Mg} / \mathrm{m}^{3}$ & \\
\hline Absorption coefficient & $1.056 \mathrm{~mm}^{-1}$ & \\
\hline$F(000)$ & 1492 & \\
\hline Crystal color, morphology & pink-black, block & \\
\hline Crystal size & $0.25 \times 0.25 \times 0.12 \mathrm{~mm}^{3}$ & \\
\hline Theta range for data collection & 1.830 to $31.522^{\circ}$ & \\
\hline Index ranges & $\begin{array}{l}-20 \leq h \leq 20,-24 \leq k \leq 24 \\
-20 \leq l \leq 20\end{array}$ & \\
\hline Reflections collected & 73096 & \\
\hline Independent reflections & $10971[R($ int $)=0.0772]$ & \\
\hline Observed reflections & 7847 & \\
\hline Completeness to theta $=31.506^{\circ}$ & $100.0 \%$ & \\
\hline Absorption correction & Multi-scan & \\
\hline Max. and min. transmission & 0.7462 and 0.6901 & \\
\hline Refinement method & $\begin{array}{l}\text { Full-matrix least-squares } \\
\text { on } F^{2}\end{array}$ & \\
\hline
\end{tabular}




\begin{tabular}{ll}
\hline Data / restraints / parameters & $10971 / 0 / 347$ \\
Goodness-of-fit on $F^{2}$ & 1.022 \\
Final $R$ indices $[I>2 \operatorname{sigma}(I)]$ & $R 1=0.0532, w R 2=0.1338$ \\
$R$ indices (all data) & $R 1=0.0793, w R 2=0.1511$ \\
Largest diff. peak and hole & 2.754 and -0.629 e. $\AA^{-3}$ \\
\hline
\end{tabular}

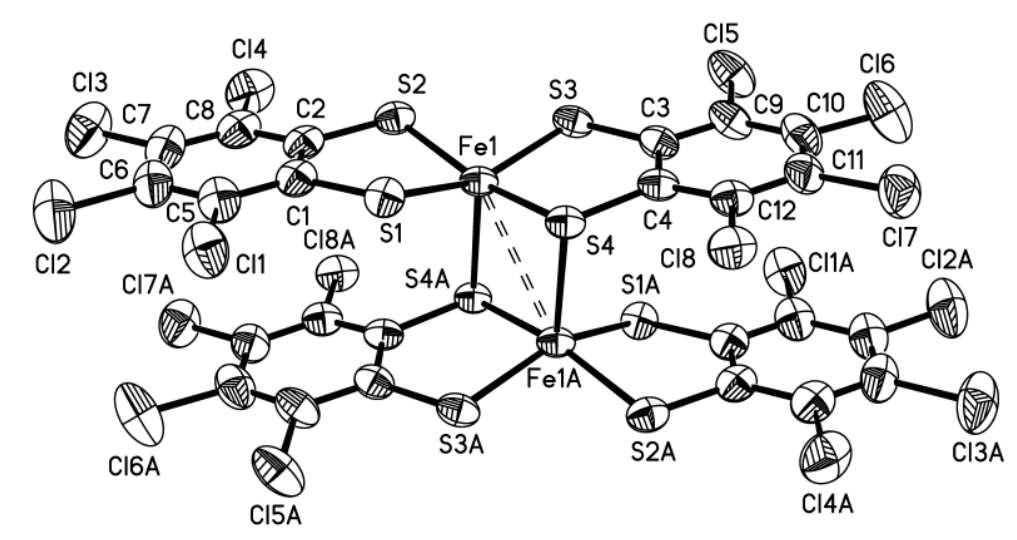

Fig. S4. ORTEP diagram of 4.

Table S4. Crystallographic data and structure refinement for $\mathbf{4}$.

\begin{tabular}{|c|c|c|}
\hline Empirical formula & $\mathrm{C}_{56} \mathrm{H}_{72} \mathrm{Cl}_{16} \mathrm{Fe}_{2} \mathrm{~N}_{2} \mathrm{~S}_{8}$ & \\
\hline Formula weight & 1708.53 & \\
\hline Temperature & $100.0(5) \mathrm{K}$ & \\
\hline Wavelength & $0.71073 \AA$ & \\
\hline Crystal system & monoclinic & \\
\hline Space group & $P 2_{1} / c$ & \\
\hline \multirow[t]{3}{*}{ Unit cell dimensions } & $a=13.9394(8) \AA$ & $\alpha=90^{\circ}$ \\
\hline & $b=18.7636(11) \AA$ & $\beta=115.9583(12)^{\circ}$ \\
\hline & $c=15.3821(9) \AA$ & $\gamma=90^{\circ}$ \\
\hline Volume & $3617.3(4) \AA^{3}$ & \\
\hline$Z$ & 2 & \\
\hline Density (calculated) & $1.569 \mathrm{Mg} / \mathrm{m}^{3}$ & \\
\hline Absorption coefficient & $1.261 \mathrm{~mm}^{-1}$ & \\
\hline$F(000)$ & 1748 & \\
\hline Crystal color, morphology & dark orange-brown, block & \\
\hline Crystal size & $0.34 \times 0.22 \times 0.14 \mathrm{~mm}^{3}$ & \\
\hline Theta range for data collection & 1.829 to $27.506^{\circ}$ & \\
\hline Index ranges & $\begin{array}{l}-18 \leq h \leq 18,-24 \leq k \leq 24 \\
-19 \leq l \leq 19\end{array}$ & \\
\hline Reflections collected & 48302 & \\
\hline
\end{tabular}


Independent reflections

Observed reflections

Completeness to theta $=27.485^{\circ}$

Absorption correction

Max. and min. transmission

Refinement method

Data / restraints / parameters

Goodness-of-fit on $F^{2}$

Final $R$ indices $[I>2 \operatorname{sigma}(I)]$

$R$ indices (all data)

Largest diff. peak and hole
$8307[R($ int $)=0.0585]$

5748

$100.0 \%$

Multi-scan

0.7456 and 0.6233

Full-matrix least-squares

on $F^{2}$

8307 / 0 / 383

1.034

$R 1=0.0574, w R 2=0.1494$

$R 1=0.0884, w R 2=0.1732$

1.121 and -0.952 e. $\AA^{-3}$

Table S5. Redox potentials for Fe-complexes (V vs SCE) determined from cyclic voltammetry.
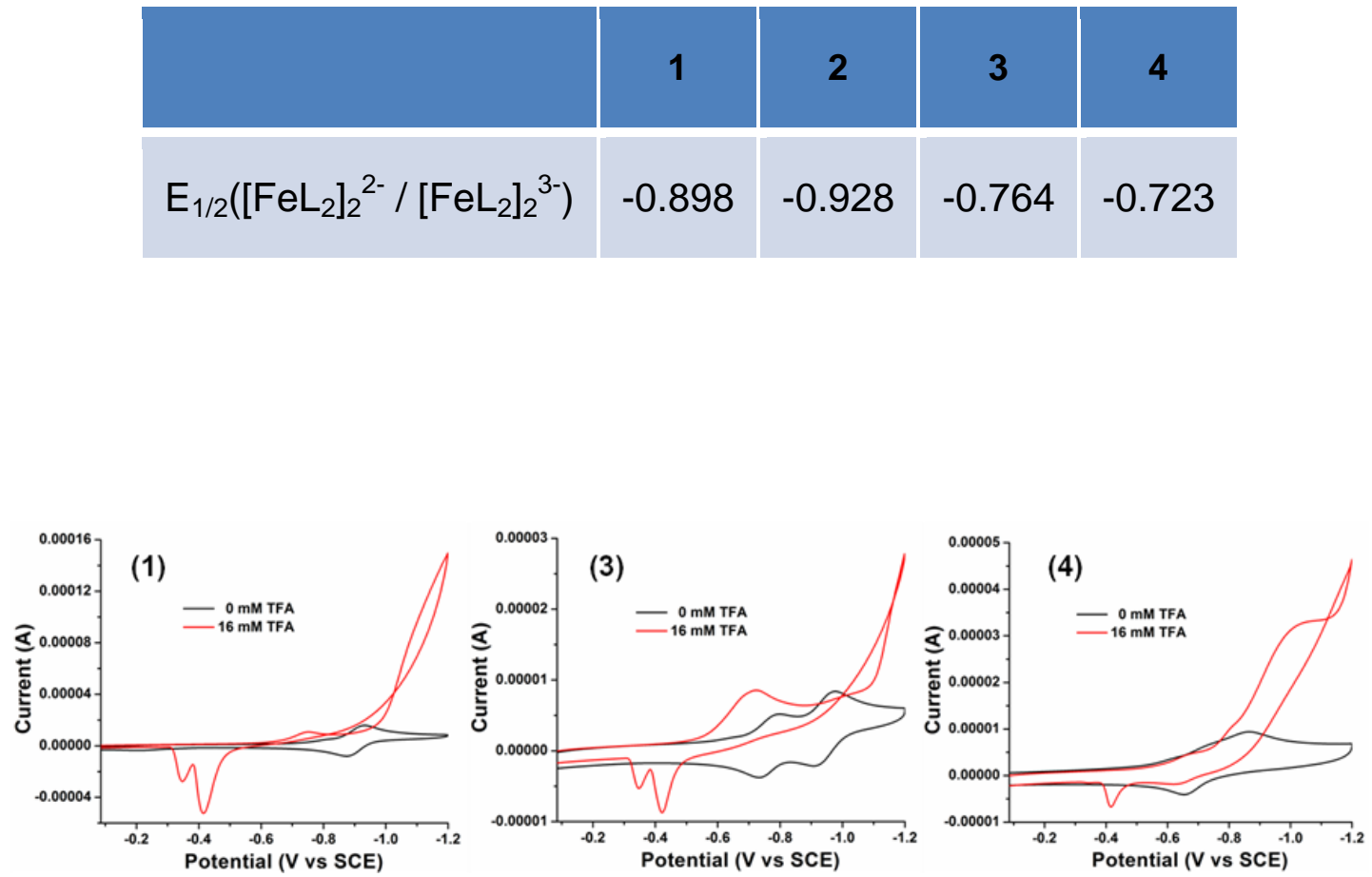

Fig. S5. Cyclic voltammograms of $0.5 \mathrm{mM}$ Fe-dithiolene complex with varying concentrations of trifluoroacetic acid (TFA). The number on each panel corresponds to the complex being examined with that of complex 2 given as Figure 4. Conditions: $0.5 \mathrm{mM}$ Fe-dithiolene complex, $0.1 \mathrm{M} \mathrm{TBAPF}_{6}$ electrolyte in acetonitrile, $1 \mathrm{M} \mathrm{H}_{2} \mathrm{O}$, glassy carbon working electrode, $\mathrm{Pt}$ auxiliary electrode, SCE reference electrode. 


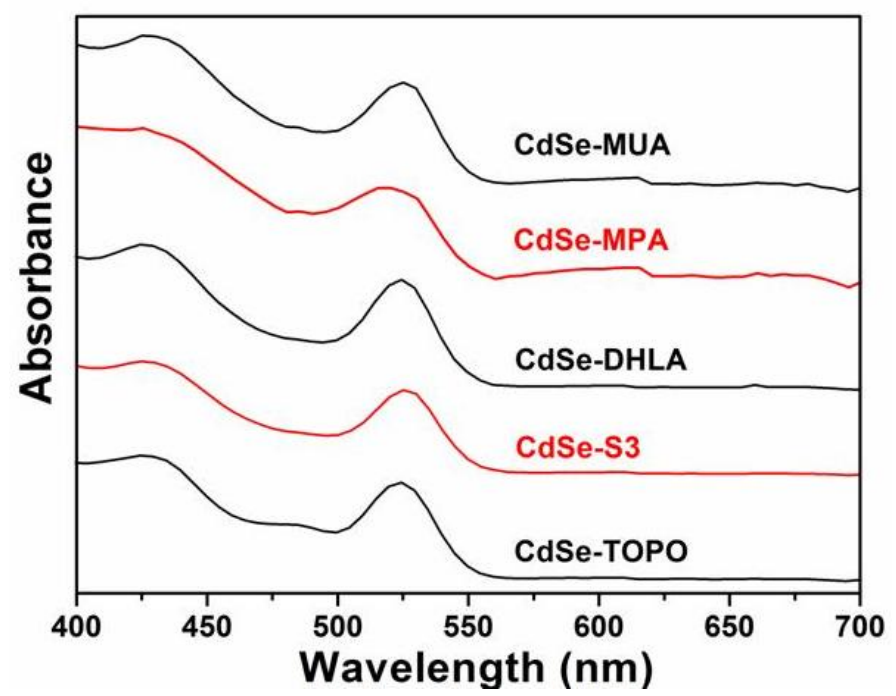

Fig. S6. UV-vis spectra of CdSe NCs with different capping agents. Note: the spectrum of CdSeTOPO was measured in hexane, while the spectra of other CdSe samples were measured in water.
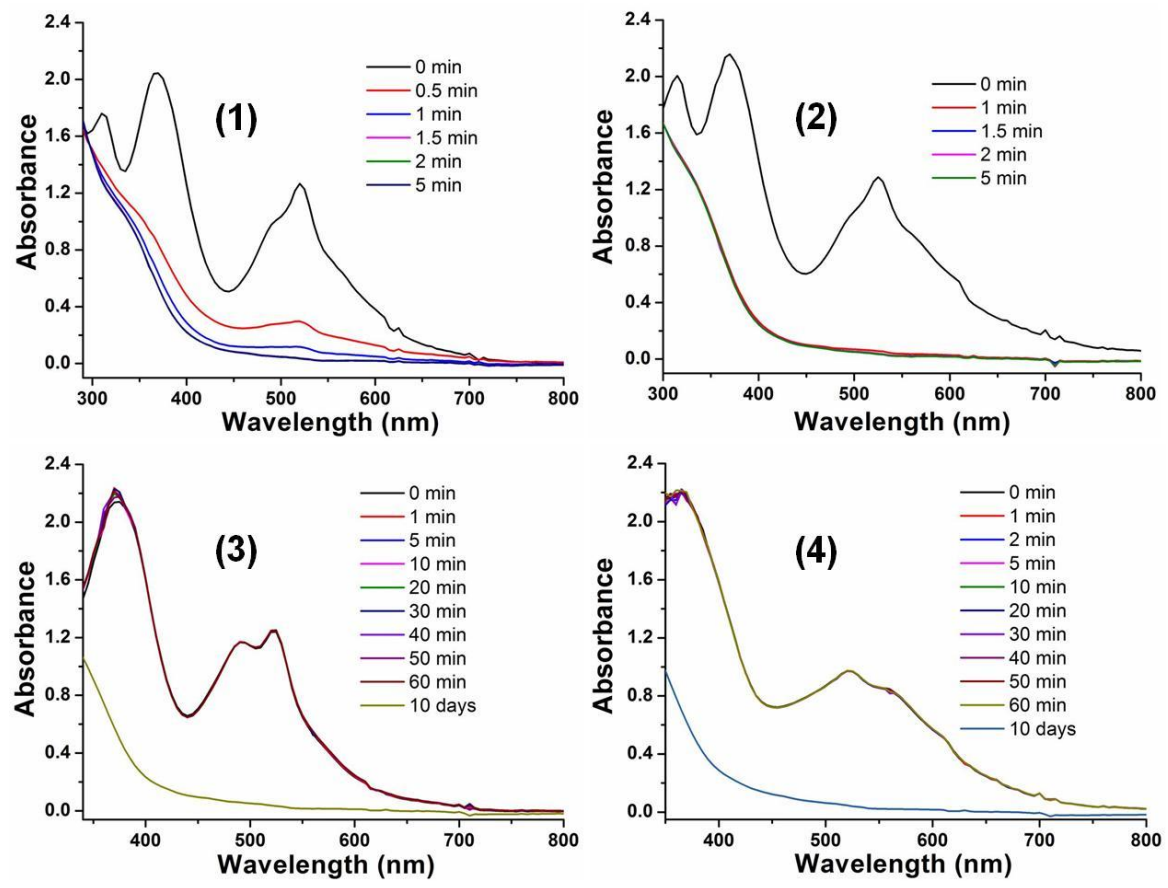

Fig. S7. UV-vis spectral change of a solution containing $0.1 \mathrm{mM}$ Fe-dithiolene complex and 4 $\mathrm{mM}$ DHLA in $\mathrm{CH}_{3} \mathrm{CN}$. The number on each panel corresponds to the complex being examined. 

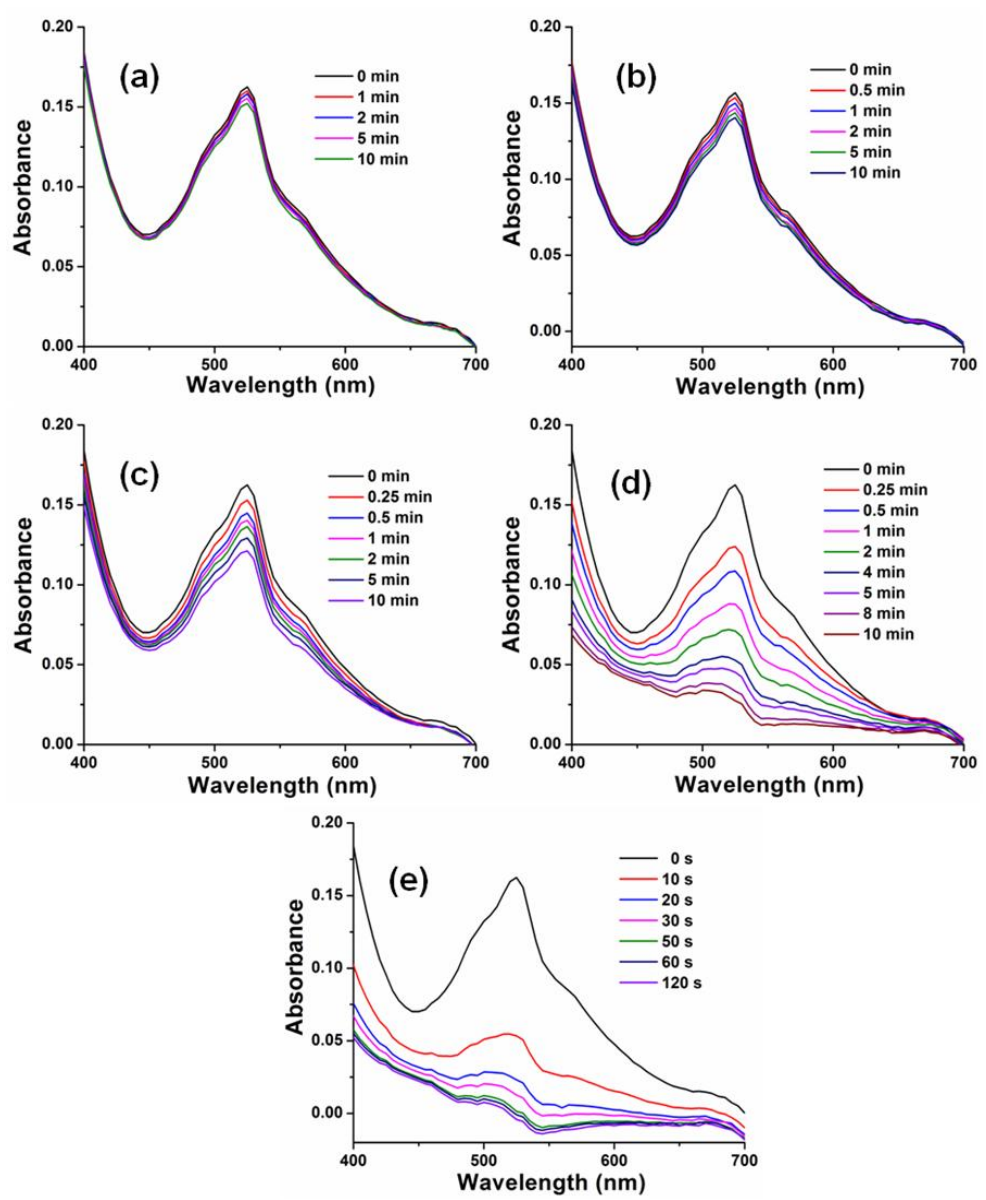

Fig. S8. UV-vis spectral change of a solution containing (a): $0.01 \mathrm{mM}$ complex $2+0.01 \mathrm{mM}$ DHLA; (b) $0.01 \mathrm{mM}$ complex $2+0.02 \mathrm{mM}$ DHLA; (c) $0.01 \mathrm{mM}$ complex $2+0.04$ mM DHLA; (d) $0.01 \mathrm{mM}$ complex $2+0.2 \mathrm{mM}$ DHLA; (e) $0.01 \mathrm{mM}$ complex $2+0.8 \mathrm{mM}$ DHLA;

Chart S1. Control experiments of hydrogen evolution (irradiation of $\sim 81 \mathrm{hrs}$ for every experiment, same reaction conditions as in Fig. 6)

\begin{tabular}{|c|c|c|c|c|}
\hline \multicolumn{4}{|c|}{ Reaction conditions } & \multirow{2}{*}{ Product $\left(\mathrm{H}_{2}\right)$} \\
\cline { 1 - 2 } $\begin{array}{c}\text { Light } \\
\text { irradiation }\end{array}$ & CdSe-S3 & AA & Complex 2 & Yes (TON = 17000 vs. per \\
\cline { 1 - 3 } Yes & Yes & Yes & Yes & little (TON = 2200 vs. QD) \\
\hline Yes & Yes & Yes & No & No \\
\hline Yes & Yes & No & Yes & No \\
\hline Yes & No & Yes & Yes & No \\
\hline No & Yes & Yes & Yes & \\
\hline
\end{tabular}




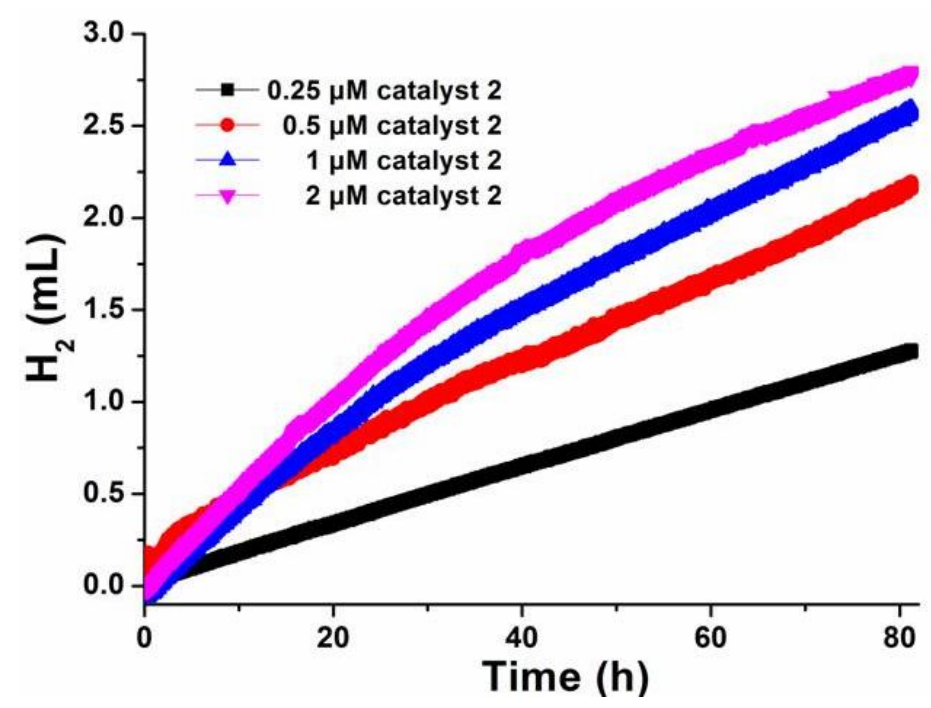

Fig. S9. Photocatalytic hydrogen production from systems containing varying concentrations of catalyst $2(0.25-2 \mu \mathrm{M})$, CdSe-S3 NCs $(515 \mathrm{~nm}, 2 \mu \mathrm{M}), \mathrm{AA}(0.2 \mathrm{M})$ in $5 \mathrm{~mL} \mathrm{EtOH} / \mathrm{H}_{2} \mathrm{O}(1 / 1)$ at $\mathrm{pH}=4.5$ upon irradiation with $520 \mathrm{~nm}$ LED at $15{ }^{\circ} \mathrm{C}$ and 1 atm initial pressure of $\mathrm{N}_{2} / \mathrm{CH}_{4}(4 / 1)$ with $\mathrm{CH}_{4}$ as an internal standard for $\mathrm{H}_{2}$ quantification by $\mathrm{GC}$ analysis.

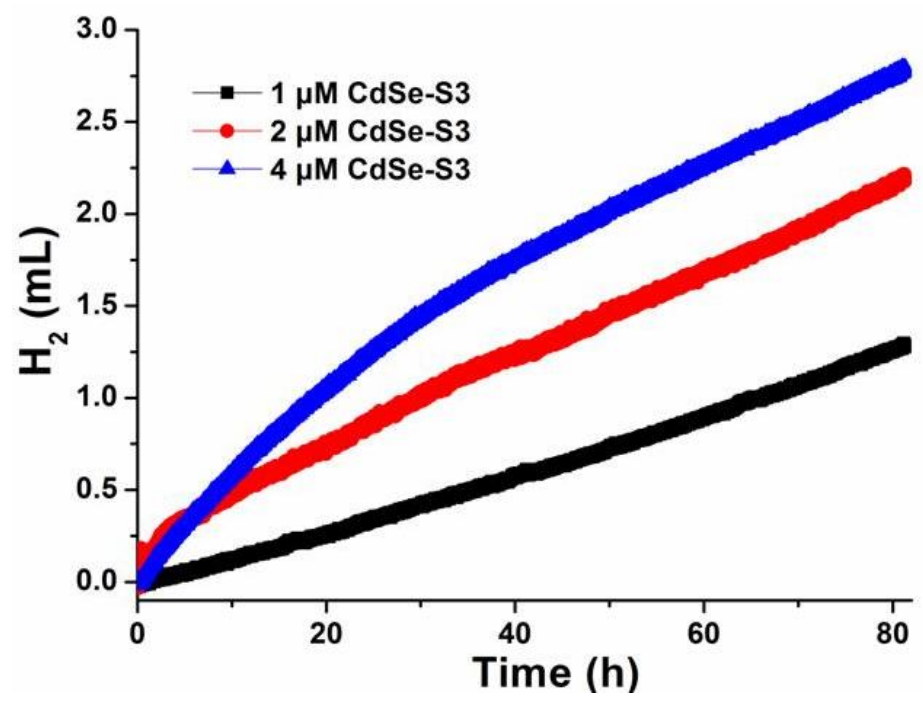

Fig. S10. Photocatalytic hydrogen production from systems containing complex $2(0.5 \mu \mathrm{M})$, varying concentrations of CdSe-S3 NCs $(515 \mathrm{~nm}, 1-4 \mu \mathrm{M}), \mathrm{AA}(0.2 \mathrm{M})$ in $5 \mathrm{~mL} \mathrm{EtOH} / \mathrm{H}_{2} \mathrm{O}$ $(1 / 1)$ at $\mathrm{pH}=4.5$ upon irradiation with $520 \mathrm{~nm}$ LED at $15{ }^{\circ} \mathrm{C}$ and 1 atm initial pressure of $\mathrm{N}_{2} / \mathrm{CH}_{4}(4 / 1)$ with $\mathrm{CH}_{4}$ as an internal standard for $\mathrm{H}_{2}$ quantification by $\mathrm{GC}$ analysis. 


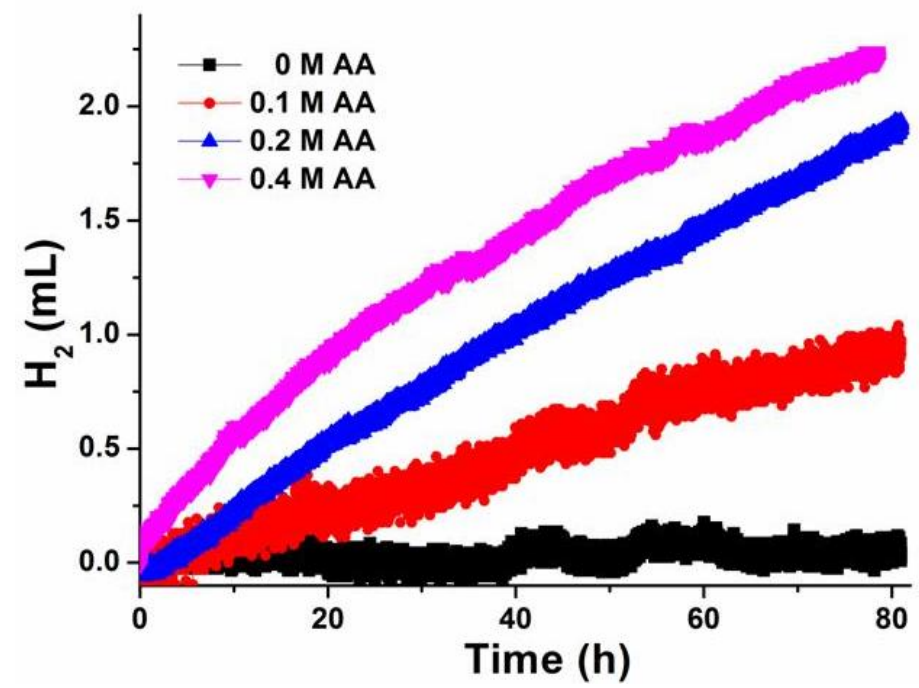

Fig. S11. Photocatalytic hydrogen production from systems containing complex $2(0.5 \mu \mathrm{M})$, CdSe-S3 NCs $(520 \mathrm{~nm}, 2 \mu \mathrm{M})$, varying concentrations of AA $(0-0.4 \mathrm{M})$ in $5 \mathrm{~mL} \mathrm{EtOH} / \mathrm{H}_{2} \mathrm{O}$ $(1 / 1)$ at $\mathrm{pH}=4.5$ upon irradiation with $520 \mathrm{~nm}$ LED at $15{ }^{\circ} \mathrm{C}$ and 1 atm initial pressure of $\mathrm{N}_{2} / \mathrm{CH}_{4}(4 / 1)$ with $\mathrm{CH}_{4}$ as an internal standard for $\mathrm{H}_{2}$ quantification by $\mathrm{GC}$ analysis.

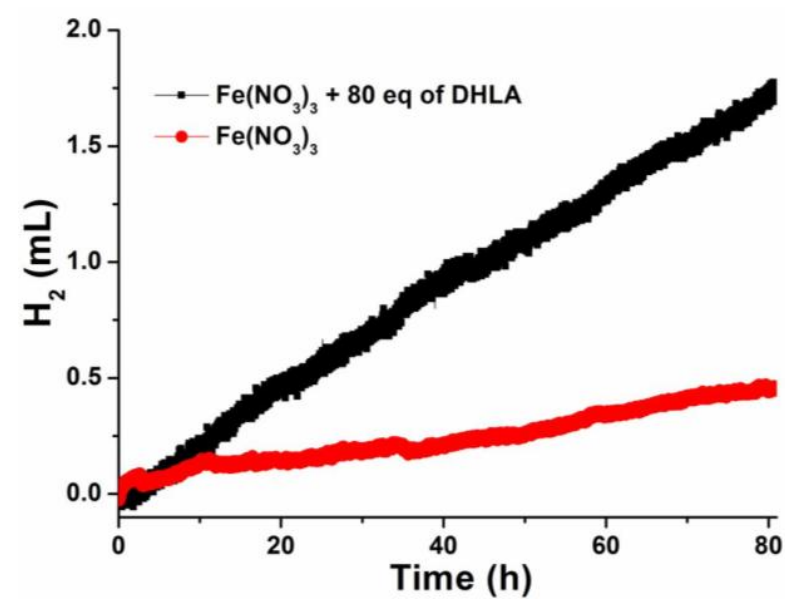

Fig. S12. Photocatalytic hydrogen production from systems containing $1 \mu \mathrm{M} \mathrm{Fe}\left(\mathrm{NO}_{3}\right)_{3}(\mathrm{red}$ curve) or $1 \mu \mathrm{M} \mathrm{Fe}\left(\mathrm{NO}_{3}\right)_{3}+80$ eq of DHLA (black curve), CdSe-S3 NCs (520 nm, $\left.2 \mu \mathrm{M}\right)$, AA $(0.2 \mathrm{M})$ in $5 \mathrm{~mL} \mathrm{EtOH} / \mathrm{H}_{2} \mathrm{O}(1 / 1)$ at $\mathrm{pH}=4.5$ upon irradiation with $520 \mathrm{~nm} \mathrm{LED}$ at $15{ }^{\circ} \mathrm{C}$ and 1 atm initial pressure of $\mathrm{N}_{2} / \mathrm{CH}_{4}(4 / 1)$ with $\mathrm{CH}_{4}$ as an internal standard for $\mathrm{H}_{2}$ quantification by $\mathrm{GC}$ analysis. 


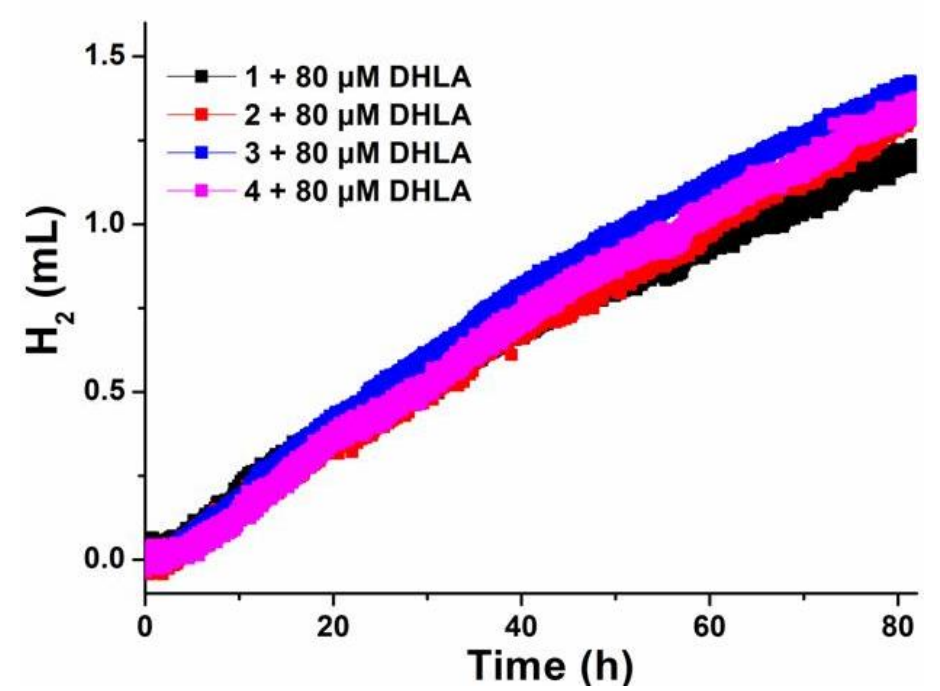

Fig. S13. Photocatalytic hydrogen production from systems containing Fe-dithiolene complexes $(0.5 \mu \mathrm{M})+$ DHLA $(80 \mu \mathrm{M}), \mathrm{CdSe}-\mathrm{S} 3 \mathrm{NCs}(520 \mathrm{~nm}, 2 \mu \mathrm{M}), \mathrm{AA}(0.2 \mathrm{M})$ in $5 \mathrm{~mL} \mathrm{EtOH} / \mathrm{H}_{2} \mathrm{O}$ $(1 / 1)$ at $\mathrm{pH}=4.5$ upon irradiation with $520 \mathrm{~nm}$ LED at $15{ }^{\circ} \mathrm{C}$ and 1 atm initial pressure of $\mathrm{N}_{2} / \mathrm{CH}_{4}(4 / 1)$ with $\mathrm{CH}_{4}$ as an internal standard for $\mathrm{H}_{2}$ quantification by $\mathrm{GC}$ analysis. The $\mathrm{Fe}-$ dithiolene complexes + DHLA reaction solution were allowed to stand for $2 \mathrm{hrs}$ prior to irradiation to allow for DHLA exchange with dithiolene ligand.

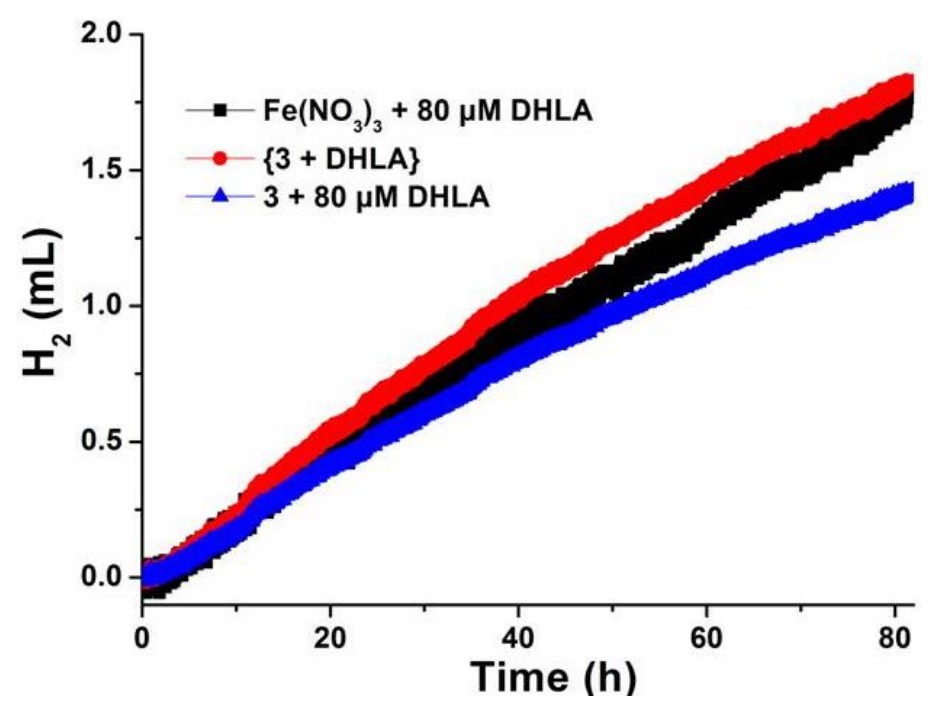

Fig. S14. Photocatalytic hydrogen production from systems containing $1 \mu \mathrm{M} \mathrm{Fe}\left(\mathrm{NO}_{3}\right)_{3}+\mathrm{DHLA}$ $(80 \mu \mathrm{M})$ (black curve) or $\{$ complex $3(0.5 \mu \mathrm{M})+$ DHLA $(40 \mu \mathrm{M})\}$ (red curve) or complex $3(0.5$ $\mu \mathrm{M})+$ DHLA $(80 \mu \mathrm{M})$ (blue curve), CdSe-S3 NCs $(520 \mathrm{~nm}, 2 \mu \mathrm{M})$, AA $(0.2 \mathrm{M})$ in $5 \mathrm{~mL}$ $\mathrm{EtOH} / \mathrm{H}_{2} \mathrm{O}(1 / 1)$ at $\mathrm{pH}=4.5$ upon irradiation with $520 \mathrm{~nm} \mathrm{LED} \mathrm{at} 15{ }^{\circ} \mathrm{C}$ and $1 \mathrm{~atm}$ initial pressure of $\mathrm{N}_{2} / \mathrm{CH}_{4}(4 / 1)$ with $\mathrm{CH}_{4}$ as an internal standard for $\mathrm{H}_{2}$ quantification by $\mathrm{GC}$ analysis. Note: the stock solution used for $\{$ complex $3(0.5 \mu \mathrm{M})+$ DHLA $(40 \mu \mathrm{M})\}$ catalysis comes from the post-solution used in Fig. S7. 
Table S6. TONs per Fe atom for $\mathrm{H}_{2}$ production using complex 2 or $\mathrm{Fe}\left(\mathrm{NO}_{3}\right)_{3}$ in the presence of varying concentrations of DHLA. Conditions: CdSe-S3 NCs $(520 \mathrm{~nm}, 2 \mu \mathrm{M})$, AA (0.2 M) in 5 $\mathrm{mL} \mathrm{EtOH} / \mathrm{H}_{2} \mathrm{O}(1 / 1)$ at $\mathrm{pH}=4.5$ upon irradiation with $520 \mathrm{~nm} \mathrm{LED}$ at $15{ }^{\circ} \mathrm{C}$ and 1 atm initial pressure of $\mathrm{N}_{2} / \mathrm{CH}_{4}(4 / 1)$ with $\mathrm{CH}_{4}$ as an internal standard for $\mathrm{H}_{2}$ quantification by $\mathrm{GC}$ analysis.

\begin{tabular}{ll}
\hline Catalyst & TON \\
\hline $\mathbf{2}(0.5 \mu \mathrm{M})$ & 17600 \\
$0.5 \mu \mathrm{M} 2+0.5 \mu \mathrm{M}$ DHLA & 19700 \\
$0.5 \mu \mathrm{M} 2+1 \mu \mathrm{M}$ DHLA & 23300 \\
$0.5 \mu \mathrm{M} 2+2 \mu \mathrm{M}$ DHLA & 15800 \\
$0.5 \mu \mathrm{M} 2+10 \mu \mathrm{M}$ DHLA & 14000 \\
$0.5 \mu \mathrm{M} 2+40 \mu \mathrm{M}$ DHLA & 13100 \\
$1 \mu \mathrm{M} \mathrm{Fe}\left(\mathrm{NO}_{3}\right)_{3}+80 \mu \mathrm{M}$ DHLA & 15300 \\
\hline
\end{tabular}

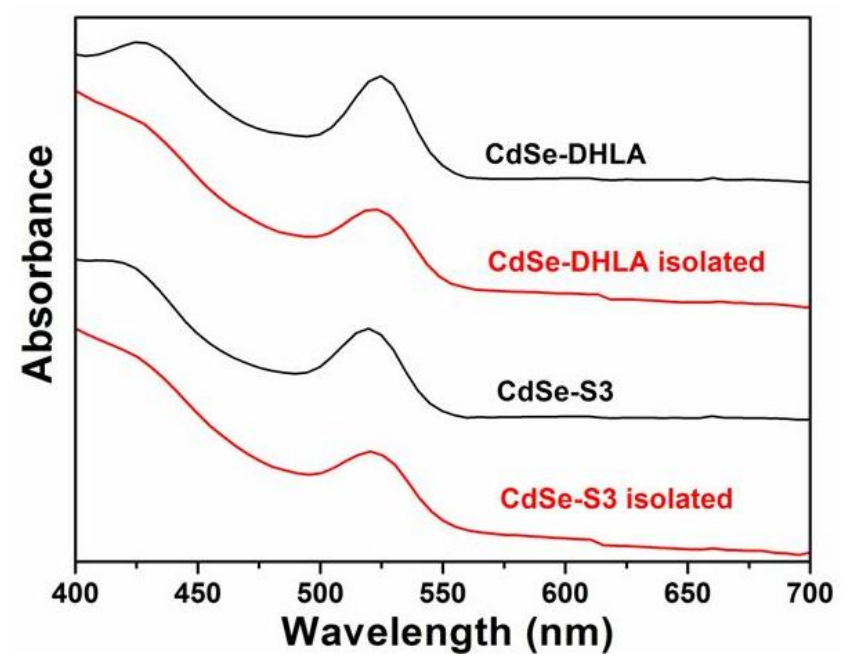

Fig. S15. UV-vis spectra of CdSe-DHLA and CdSe-S3 NCs before and after photolysis. 


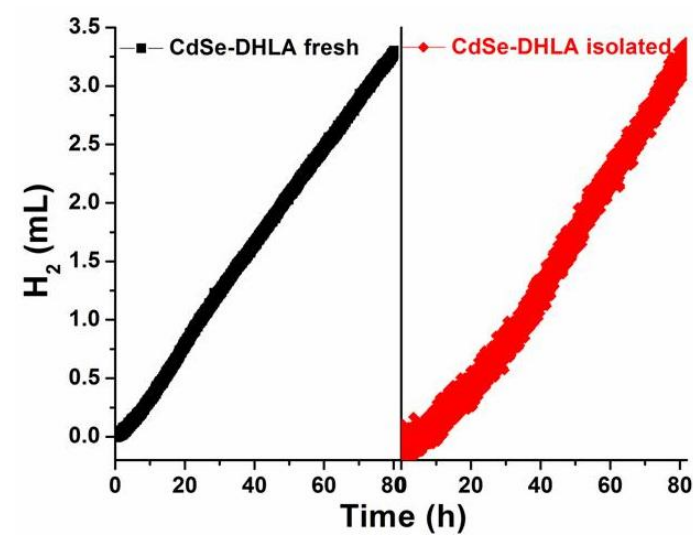

Fig. S16. Photocatalytic hydrogen production from systems containing complex $2(0.5 \mu \mathrm{M})$, fresh CdSe-DHLA NCs $(525 \mathrm{~nm}, 2 \mu \mathrm{M}$; black curve) or isolated CdSe-DHLA NCs (524 nm, 2 $\mu \mathrm{M}$; red curve), $\mathrm{AA}(0.2 \mathrm{M})$ in $5 \mathrm{~mL} \mathrm{EtOH} / \mathrm{H}_{2} \mathrm{O}(1 / 1)$ at $\mathrm{pH}=4.5$ upon irradiation with $520 \mathrm{~nm}$ LED at $15{ }^{\circ} \mathrm{C}$ and $1 \mathrm{~atm}$ initial pressure of $\mathrm{N}_{2} / \mathrm{CH}_{4}$ (4/1) with $\mathrm{CH}_{4}$ as an internal standard for $\mathrm{H}_{2}$ quantification by GC analysis.

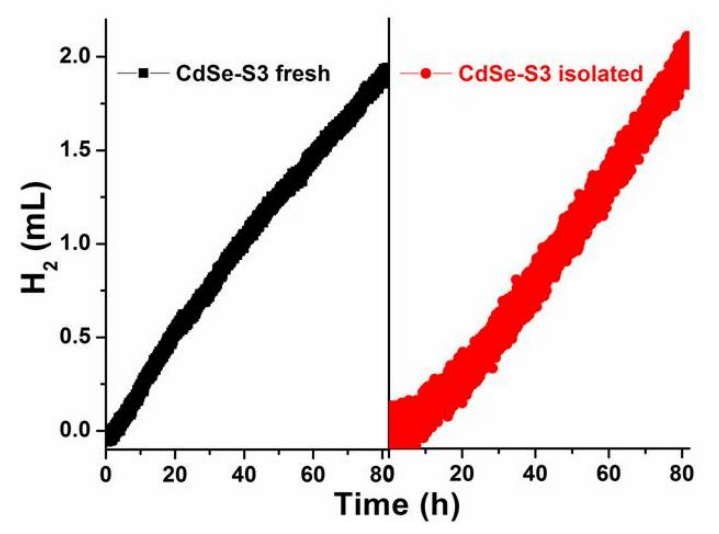

Fig. S17. Photocatalytic hydrogen production from systems containing complex $2(0.5 \mu \mathrm{M})$, fresh CdSe-S3 NCs $(520 \mathrm{~nm}, 2 \mu \mathrm{M}$; black curve) or isolated CdSe-S3 NCs $(520 \mathrm{~nm}, 2 \mu \mathrm{M}$; red curve), $\mathrm{AA}(0.2 \mathrm{M})$ in $5 \mathrm{~mL} \mathrm{EtOH} / \mathrm{H}_{2} \mathrm{O}(1 / 1)$ at $\mathrm{pH}=4.5$ upon irradiation with $520 \mathrm{~nm}$ LED at $15{ }^{\circ} \mathrm{C}$ and 1 atm initial pressure of $\mathrm{N}_{2} / \mathrm{CH}_{4}$ (4/1) with $\mathrm{CH}_{4}$ as an internal standard for $\mathrm{H}_{2}$ quantification by GC analysis.

Table S7. TONs per Fe atom for $\mathrm{H}_{2}$ production using different $\mathrm{Fe}$ dithiolene catalysts and $\mathrm{CdSe}-$ S3 QDs with different amounts of S3 capping agent.

\begin{tabular}{rrrc}
\hline Catalyst & $\begin{array}{c}\text { CdSe-S3 } \\
(\mathbf{7 0} \text { units, } \mathbf{5 2 3} \mathbf{~ n m})\end{array}$ & $\begin{array}{c}\text { CdSe-S3 } \\
(\mathbf{1 0 0} \text { units, } \mathbf{5 2 0} \mathbf{~ n m})\end{array}$ & $\begin{array}{c}\text { CdSe-S3 } \\
(\mathbf{1 8 0} \text { units, } \mathbf{5 1 5} \mathbf{~ n m})\end{array}$ \\
\hline $\mathbf{1}$ & 14300 & 11100 & 3400 \\
$\mathbf{2}$ & 20700 & 17600 & 4600 \\
$\mathbf{3}$ & 11500 & 9100 & 2400 \\
$\mathbf{4}$ & 9500 & 8400 & 2200 \\
\hline
\end{tabular}




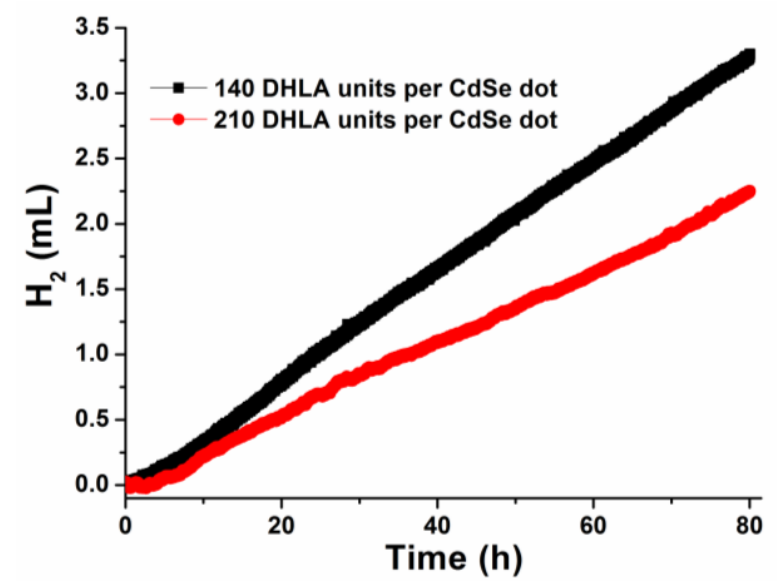

Fig. S18. Photocatalytic hydrogen production from systems containing complex $2(0.5 \mu \mathrm{M}), 2$ $\mu \mathrm{M}$ CdSe-DHLA NCs (black curve: $525 \mathrm{~nm}$ with 140 DHLA units; red curve: $520 \mathrm{~nm}$ with 210 DHLA units), AA $(0.2 \mathrm{M})$ in $5 \mathrm{~mL} \mathrm{EtOH} / \mathrm{H}_{2} \mathrm{O}(1 / 1)$ at $\mathrm{pH}=4.5$ upon irradiation with $520 \mathrm{~nm}$ LED at $15{ }^{\circ} \mathrm{C}$ and 1 atm initial pressure of $\mathrm{N}_{2} / \mathrm{CH}_{4}$ (4/1) with $\mathrm{CH}_{4}$ as an internal standard for $\mathrm{H}_{2}$ quantification by $\mathrm{GC}$ analysis.

Table S8. TONs for hydrogen production using different Fe-catalysts and CdSe-DHLA NCs

\begin{tabular}{ccc}
\hline Catalyst & $\begin{array}{c}\text { CdSe-DHLA } \\
(\mathbf{1 4 0} \text { units, 525 } \mathbf{~ n m})\end{array}$ & $\begin{array}{c}\text { CdSe-DHLA } \\
(\mathbf{2 1 0} \text { units, 520 } \mathbf{~ m})\end{array}$ \\
\hline $\mathbf{1}$ & 20600 & 13600 \\
$\mathbf{2}$ & 29400 & 20100 \\
$\mathbf{3}$ & 15200 & 10300 \\
$\mathbf{4}$ & 8000 & 7300 \\
\hline
\end{tabular}

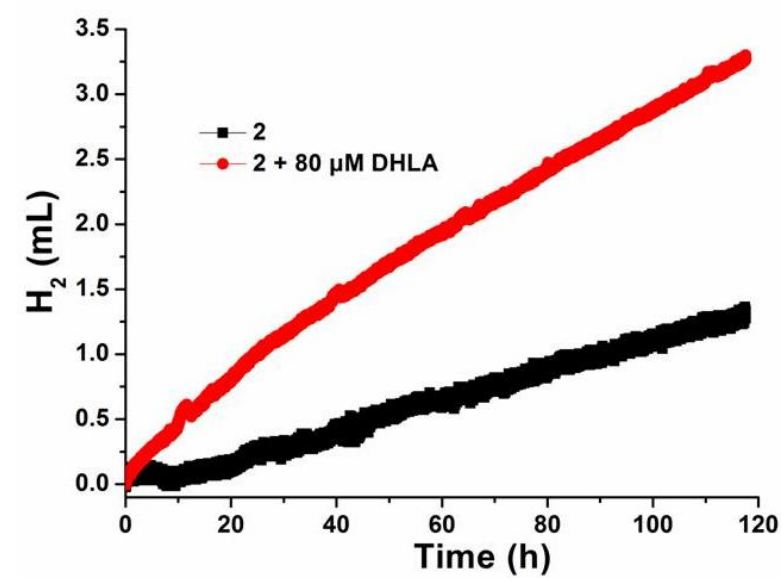

Fig. S19. Photocatalytic hydrogen production from systems containing CdSe-MUA NCs (525 nm, $1 \mu \mathrm{M})$, complex $2(0.5 \mu \mathrm{M}$; black curve) or complex $2(0.5 \mu \mathrm{M})+$ DHLA $(80 \mu \mathrm{M})$ (red curve), $\mathrm{AA}(0.2 \mathrm{M})$ in $5 \mathrm{~mL} \mathrm{EtOH} / \mathrm{H}_{2} \mathrm{O}(1 / 1)$ at $\mathrm{pH}=4.5$ upon irradiation with $520 \mathrm{~nm} \mathrm{LED}$ at $15{ }^{\circ} \mathrm{C}$ and $1 \mathrm{~atm}$ initial pressure of $\mathrm{N}_{2} / \mathrm{CH}_{4}$. 


\section{References}

(1) Han, Z.; Qiu, F.; Eisenberg, R.; Holland, P. L.; Krauss, T. D. Science 2012, 338, 1321.

(2) Das, A.; Han, Z.; Haghighi, M. G.; Eisenberg, R. Proc. Natl. Acad. Sci. USA 2013, 110, 16716.

(3) Aldana, J.; Lavelle, N.; Wang, Y.; Peng, X. J. Am. Chem. Soc. 2005, 127, 2496. 